Christine RogL

\title{
ZU DEM PRODUKTIONSBEGINN SCHWARZER SIGILLATA IN EPHESOS \\ Die Evidenz der Tetragonos Agora
}

Von der schwarzen Sigillata, einer lokal ephesischen Keramikgattung, wurden bislang vor allem die sog. grauen Ephesos-Platten in der archäologischen Literatur bekanntgemacht. Diese stellen den meistverwendeten terminus technicus in bezug auf die Gattung dar und fanden auch offensichtlich als ihre Hauptform - die weiteste Verbreitung im Mittelmeerraum. Im Folgenden soll eine Definition der Gattung und ihrer bisher aus Ephesos bekannten frühen Formen versucht werden.

\section{Erscheinungsbild}

Ton- und Firnisqualitäten der in die Diskussion einbezogenen Stücke entsprechen im Detail jenen der Ephesos-Platten ${ }^{1}$, d. h., ihr Ton erscheint grau oder graubraun und enthält Hellglimmer, Kalkpartikel und Kalkpseudomorphosen sowie Quarz, Feldspat, Spuren von Schwermineralien und Bioklasten. Sie sind hart gebrannt, bei nicht allzu hohen Temperaturen, und wirken im Bruch körnig, manchmal auch porös. Der Überzug changiert zwischen dunklem Grau und tiefem Schwarz und erweckt einen polierten bzw. glasartigen Eindruck an der Oberfläche. Dieser glänzende Effekt verliert sich nur bei stark abgenutzten Stellen bzw. an der Gefäßunterseite, falls diese nicht teilweise, späthellenistischer Tradition folgend, ungefirnißt geblieben ist. Die Wandungen sind massiv und rund gebildet.

Von den hier präsentierten Beispielen der schwarzen ephesischen Sigillata sind jene der sog. grauen Keramik bzw. grauen Ware mit eher mattem und trockenem Firnis, z. B. graue Firniskeramik, graue Reliefbecher, graue Ephesos-Lampen, graue Figuralgefäße etc., welche ebenfalls in Ephesos hergestellt wurden, zu unterscheiden. Bei chemischen Analysen der Tonzusammensetzung der genannten Gattungen fielen bisher alle in eine engbegrenzte Gruppe; die petrographischen Analysen zeigten allerdings deutliche Unterschiede im Brenngrad, auch die Schwermineralzusammensetzung läßt im Detail eine Trennung der Grauen Platten von den Reliefbechern und Ephesos-Lampen $\mathrm{zu}^{2}$. Die archäologische Bearbeitung kann hier zusätzlich auf eindeutige Unterschiede im Oberflächencharakter, in der Oberflächengestaltung und im jeweiligen Formenrepertoire zwischen der schwarzen ephesischen Sigillata und den graugebrannten griechischhellenistischen Gattungen der Firniskeramik hinweisen.

Somit kann aufgrund des beschriebenen Erscheinungsbildes diese Keramikgattung allgemein den frühesten Sigillaten zugeordnet werden. Ihre Beziehung zu den frühen östlichen und westlichen Sigillaten drückt sich auch in den Ähnlichkeiten ihrer Formen aus, wie unten noch auszuführen sein wird.

\footnotetext{
1 Von Ph. Kenrick 1985, 52 als »Black-glazed $\mathrm{C}^{2}$-ware« bezeichnet und in ihren Eigenschaften beschrieben. Vgl. dazu auch Mitsopoulos-Leon 1991, 78; Outschar 2000, 142 und bes. Zabehlicky-Scheffenegger - Sauer - Schneider 1996, 48-49 mit Tab. 1-2 auf S. 51. - Die hier verwendeten Abkürzungen und die abgekürzt zitierte Literatur finden sich am Ende des Beitrags.

2 Vgl. Zabehlicky-Scheffenegger - Sauer - Schneider 1996, 49. 54.
} 


\section{Formen}

Aufgrund der bisherigen archäologischen Befunde in Ephesos soll versucht werden, die frühesten Formen dieser lokalen Sigillata zu eruieren ${ }^{3}$ - und damit auch den Beginn einer Sigillataproduktion in Ephesos bzw. im Umland. Ausgangspunkt sind dabei die Grabungen im Bereich der hellenistischen Westhallen auf der Tetragonos Agora mit den umfangreichen Scherbenansammlungen aus den sog. Raubgruben (besonders Fundkiste 93/32) und dem sehr kleinteiligen Keramikmaterial der oberen Straßenhorizonte ${ }^{4}$. Es handelt sich um die spätesten hellenistischen Befunde, die in diesem Bereich ergraben werden konnten; in ihnen finden sich erstmals Fragmente der schwarzen Sigillata 5 . Der Fundkontext läßt eine Datierung in bzw. an das Ende des dritten Viertels des 1. Jahrhunderts v. Chr. zu. Die Fragmente sind kleinteilig, ihre Bruchflächen stark abgerundet und verschmutzt, die verglaste Oberfläche ist mitunter stark verrieben und wirkt dadurch matt ${ }^{6}$. Folgende Formen lassen sich erschließen:

- Runde Platte mit niedrigem gerundetem Rand und einfachem Ringfuß

- Runde Platte mit hohem gerundetem Rand

- Runde Platte oder Schüssel mit hohem, außen gerilltem Rand

- Runde Platte oder Schüssel mit hohem ausschwingendem Rand

- Runde Platte oder Schüssel mit hohem, weitausladendem massivem Rand

- Runde Platte mit breitem horizontal abgesetztem Rand

- Runde(?) Platte mit breitem horizontalem Rand

- Halbkugelförmiges Schälchen auf leicht profiliertem Ringfuß.

Weitere frühe Formen sind derzeit nicht bekannt ${ }^{7}$. Der Vollständigkeit halber soll noch auf ein bisher als pilzförmig angesprochenes Gefäßfragment aus Ephesos ${ }^{8}$ hingewiesen werden, dessen komplette ursprüngliche Form ebenfalls nicht mit Gewißheit erschlossen werden kann.

3 Versuche einer Erstellung von Formentwicklung und Chronologie bei Zabehlicky-Scheffenegger - Sauer - Schneider 1996, 45. Dabei sind zwei Hauptformen zu unterscheiden: die runden auf der Töpferscheibe hergestellten Platten mit niedrigem Standring und die rechteckigen modelgeformten Platten ohne Standring, jedoch oft mit zwei Griffen. Eine vorläufige Festlegung der Plattenproduktion in Ephesos wurde für das 1. Jh. v. Chr. bis in die ersten Jahrzehnte des 1. Jhs. n. Chr. vorgeschlagen.

$4 \mathrm{Zu}$ den Grabungen und der vorläufig erarbeiteten Stratigraphie s. P. Scherrer, ÖJh 63, 1994, Beibl. Grabungen 11-14; ders., ÖJh 65, 1996, Beibl. Grabungen 7-12; ders., ÖJh 66, 1997, Beibl. Grabungen 4-6; ders., ÖJh 67, 1998, Beibl. Grabungen 7-10.

5 Für diese kann aufgrund der Fundvergesellschaftung ein Schlußdatum in die späteste hellenistische Zeit vorgeschlagen werden. Neben älterem Keramikmaterial findet sich zahlreich frisch gebrochene ESA (Hayes 1985, Form 4A, 22A/B, 9-12, 13B und 14; Mitsopoulos-Leon 1975, 103 Abb. 1 und 105 Abb. 2 mit den genau übereinstimmenden ESA-Formen und einer Datierung dieser in das 3. Viertel des 1. Jhs. v. Chr.), erste einfache ESB-Schälchen (Hayes 1985, Form 14) und dünnwandige Keramik sowie zahlreiche Ephesos-Lampen. Vgl. die Chronologiediskussion bei Ch. Rogl, Späthellenistische Applikenkeramik und Verwandtes aus Ephesos, ÖJh 72, 2003, 187-188 mit Anm. 3. Zu beachten sind auch die enthaltenen Tintenfäßchen. Vgl. Ph. Zapheiropoulos in: L. Kypraiou (Hrsg.), Hellenistic pottery from the Aegean (1994) 75 Abb. 2 mit einer Datierung um 40 v. Chr.

6 Sie weisen im Vergleich zu der in großen Fragmenten erhaltenen, eindeutig frisch gebrochenen ESA einen älteren Charakter auf. Zur Datierung der ESA-Formen s. Anm. 5.

7 Jene Gefäßformen, welche von U. Outschar, vgl. Outschar 2000, 114. 142-144 Abb. 15 Kat. 111-116, an die Fabrikatsgruppe der Grauen Platten angeschlossen wurden, sind der Autorin bisher nur als Vertreter der üblichen graugebrannten ephesischen Firniskeramik bekannt. Dies gilt teilweise auch für Outschar 1996, 42-44.

8 Mitsopoulos-Leon 1991, 81. 85 Taf. 104, F 63. Vgl. dazu P. Chatzidakis in: 4. Kongreß zur hellenistischen Keramik, Mytilene 1994 (1997) 305 mit Anm. 60-61 Taf. 227. 228a-c mit ähnlichen Gefäßfragmenten, welche er als AskoiThermophores anspricht. Diese tragen auch Signaturen. Aufgrund der Beschreibung könnten sie der reduzierend gebrannten ESA bzw. einmal der schwarzen ephesischen Sigillata zugerechnet werden. Die Form an sich erinnert an die aus der Spätantike bekannten Pilgerflaschen; Flaschen oder Behältnisse in dieser Form finden sich aber bereits in hellenistischer Zeit. Vgl. Chatzidakis a. O.; E. Basiotopoulou-Valavani, Hellenistische Keramik aus Kallipolis (Ätolien), in: 3. Kongreß zur hellenistischen Keramik, Thessaloniki 1991 (1994) 54 Taf. 23. 


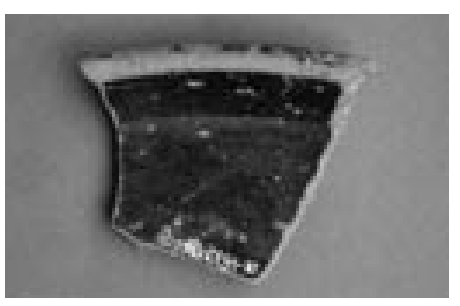

$1 \quad$ K 1. Runde Platte mit niedrigem gerundetem Rand und einfachem Ringfuß

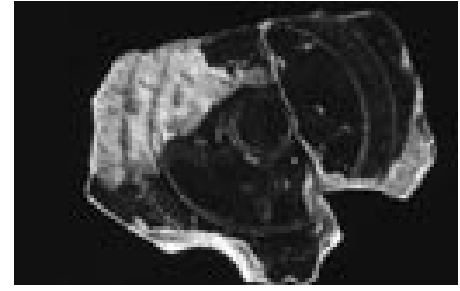

2 K 7. Palmettendekor und Roulettierung auf der Innenseite einer runden Platte

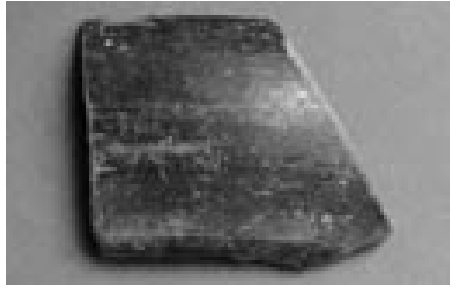

3 K 10. Runde Platte mit hohem gerundetem Rand

Die noch unpublizierten Funde aus Samos, der Ephesos vorgelagerten Insel, ergänzen hingegen nur das hier vorgestellte Repertoire der runden Platten und tiefen Schüsseln?

Aufgrund der bisherigen Fundsituation - die späthellenistische Zeit und den Übergang zur frührömischen Epoche betreffend - können die runden Platten mit niedrigem Rand als die häufigste Form dieser Gattung angesehen werden. Jene mit hohem gerundetem Rand sind bereits selten anzutreffen, und alle anderen hier gezeigten Beispiele der Schüsseln und Schalen erweisen sich als Einzelstücke bzw. Sonderformen; dies gilt nicht nur für die Fundsituation im Bereich der Tetragonos Agora, sondern findet auch Bestätigung in den Befunden der übrigen Fundplätze von Ephesos und Samos.

\section{Beschreibung der Formen}

Die runden Platten mit niedrigem gerundetem Rand und einfachem Ringfuß ${ }^{10}$ (KatNr. 1-9; Taf. 1 und Abb. 1) zeichnen sich durch weitere Details wie eine Lippe mit gerundetem Verlauf (KatNr. 1. 2) bzw. eine Lippe mit einem eher dreieckigen Querschnitt aus (KatNr. 3-6); letztes ist häufiger zu finden. Die Höhe der Lippe kann 2-2,3 cm erreichen. Der flache Boden kann nach außen hin leicht ansteigen. Der obere Randdurchmesser liegt meist um die $30 \mathrm{~cm}$, bei den hier besprochenen Beispielen liegt er zwischen ca. 25 und $37 \mathrm{~cm}$, andere ephesische Belege erreichen einen Durchmesser von bis zu $50 \mathrm{~cm}^{11}$. Der Ringfuß kann eher flach und mit einer Kehlung außen versehen, aber auch blockförmig und etwas höher gebildet sein. Der Ringfußdurchmesser beträgt zwischen 8 und $11 \mathrm{~cm}^{12}$. Die meisten dieser Platten tragen einen Dekor am inneren Plattenboden. Dabei handelt es sich um konzentrische Kreisrillen, welche einerseits mit roulettiertem Banddekor gefüllt sind und andererseits in den größeren Zwischenräumen Platz für Palmettenstempel unterschiedlichster Variation bieten. Hier sind meist vier bis fünf Palmetten um einen oder zwei Mittelkreise angeordnet. KatNr. 7-9 (Abb. 2) zeigen diese teils stark stili-

\footnotetext{
9 Freundliche Mitteilung von S. Morhoff (München), Bearbeiterin der hellenistischen Keramik auf Samos. Die Ränder der Platten erreichen eine Höhe zwischen 1,6 und 3,2 cm; ein Schüsselfragment mit Rillen auf dem Rand besitzt einen Durchmesser von ca. $32 \mathrm{~cm}$. Ton, Überzug und Wandstärke der Stücke entsprechen jenen der ephesischen Produkte.

10 Mitsopoulos-Leon 1991, 79-80. 82. 84 Taf. 94, F 2-7. 9-13; 98-100, F 44-48; Outschar 1996, 42-44 Kat. 70(?). 71. 73. 77. 80-85. 90-91; Outschar 2000, 142 Abb. 15 Kat. 109-110; Meriç 2002, 39 Taf. 11, K 97-100; Taf. 12, K 111 und S. 45 Taf. 17, K 153 (die Benennung als italische schwarze Sigillata ist anzuzweifeln), alle aus Füllung A; Ladstätter u. a. 2003, 24 Taf. 1, K 9-10; Zabehlicky-Scheffenegger - Sauer - Schneider 1996, $42-43$ Taf. 11, 2. 11; Taf. 18, 57, vielleicht auch Nr. 58. Ihre Nr. 59-60 besitzen bereits stärker profilierte Lippen (wie Meriç 2002, Taf. 11, K 101). - Sollte diese Profilierung bereits als Hinweis auf eine etwas spätere Entwicklung in Richtung niedriger profilierter Wulstrand und somit flacher Platte bzw. Tablett gedeutet werden?

11 Mitsopoulos-Leon 1991, 82 Taf. 94, F 13; Outschar 2000, 142 Abb. 15 Kat. 110. Outschar 1996, 42 mit Anm. 168 verweist auf eine solche Platte mit 1,2 m DmR im Efes Müzesi Selçuk.

12 Mitunter kommen auch größere vor. Vgl. Meriç 2002, 41, K 111 mit einem DmB von 13 cm.
} 


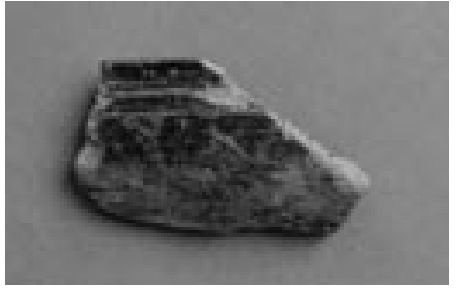

4 K 12. Runde Platte oder Schüssel mit hohem, außen gerilltem Rand

sierten, mitunter aber auch noch sehr naturalistischen Palmetten. KatNr. 6 besitzt eine stilisierte Blattpalmette mit Voluten, die den häufig erscheinenden Isiskronen-Motiven leicht ähnelt. Andere bereits veröffentlichte Beispiele aus Ephesos ergänzen dieses Repertoire um einige Varianten und um das Thyrsosmotiv $^{13}$.

Die runden Platten mit hohem gerundetem Rand ${ }^{14}$ (KatNr. 10-11; Taf. 2 und Abb. 3) werden durch einen hohen, schmalen und leicht nach außen gekippten Rand gekennzeichnet. Die Außenseite des Randes ist dabei stärker gewölbt. Seine Höhe kann mit knapp unter $5 \mathrm{~cm}$ angegeben werden. Die Maße des Randdurchmessers dürften denen der Platten mit niedrigem Rand entsprechen. KatNr. 10 läßt auf einen Durchmesser von $26 \mathrm{~cm}$ und KatNr. 11 auf $34 \mathrm{~cm}$ schließen. Das Beispiel aus der Basilika am Staatsmarkt erweist sich mit $38 \mathrm{~cm}$ Randdurchmesser als das größte. Ob diese Gefäße mit einem Ringfuß oder einem Standring und mit Verzierungen versehen waren, kann anhand der drei derzeit bekannten Exemplare und aufgrund ihres Erhaltungszustandes nicht überprüft werden. Die im Ansatz erhaltenen Plattenböden von KatNr. 10 und 11 scheinen im Vergleich jedenfalls etwas dünnwandiger zu sein. Die Form dieser Platten erinnert an die sog. pompejanisch-roten Platten ${ }^{15}$.

Von den runden Platten oder Schüsseln mit hohem, außen gerilltem Rand (KatNr. 12; Abb. 4) ist der obere Teil des leicht gewölbten Randes eines tieferen Gefäßes mit $27 \mathrm{~cm}$ Durchmesser erhalten. Im oberen Bereich der Außenseite befinden sich zwei tiefe Rillen. Es läßt sich nicht erschließen, ob sich die Wölbung fortsetzt oder ein flacher Boden folgt. Vergleichsbeispiele in derselben Qualität fehlen bisher; es finden sich auch sonst keine Parallelen ${ }^{16}$.

Von den runden Platten oder Schüsseln mit hohem ausschwingendem Rand (KatNr. 13) besitzen wir nur den Rand einer tiefen Platte oder Schüssel, über deren weiteren Formverlauf derzeit keine Aussage getroffen werden kann. Mit $36 \mathrm{~cm}$ Randdurchmesser nimmt sie die üblichen Maße dieser Gattung auf.

Von der Existenz tiefer Schüsseln oder Becken mit hohem, weit ausladendem massivem Rand zeugt das große Randfragment KatNr. 14. Weitere Einzelheiten bleiben uns aber auch hier verschlossen; der Randdurchmesser von $50 \mathrm{~cm}$ entspricht den Servicemaßen.

Das kleine Randfragment einer runden Platte mit breitem horizontal abgesetztem Rand (KatNr. 15) könnte zu einer flachen bzw. tieferen runden Platte ergänzt werden. Der Randdurchmesser ist nicht mehr eindeutig zu bestimmen, liegt aber zwischen 30 und $38 \mathrm{~cm}$.

Bei einem weiteren Fragment (KatNr. 16) läßt der Erhaltungszustand ebenfalls keine Schlüsse auf nähere Details zu. Es könnte sich um eine flache runde Platte mit breitem horizontalem Rand handeln, deren massive Wandstärke auf eine entsprechende Größe hinweist.

Von einem halbkugelförmigen Schälchen auf leicht profiliertem Ringfuß (KatNr. 17) ${ }^{17}$ hat sich teils der Ringfuß mit einer Außenkehlung, teils die Wandung erhalten. Im Inneren ist eine schmale Roulettierung angebracht. Gut vergleichbar ist ein grautoniges halbkugelförmiges Schäl-

13 Vgl. auch Zabehlicky-Scheffenegger - Sauer - Schneider 1996, 44 Taf. 11, 11; Meriç 2002, Taf. 11, K 97; Taf. 12, K 111-112; 17, K 153; Mitsopoulos-Leon 1991, 79-80 Taf. 98-100, F 42-48. - In Athen fand sich eine EphesosPlatte in einem Kontext des 1. Jhs. v. Chr., welche mit einer ionischen Säule als Stempelmotiv im Zentrum dekoriert ist, vgl. Rotroff 1997, 233. 399 Abb. 75 Nr. 1574.

14 Mitsopoulos-Leon 1991, 79. 82 Taf. 94, F 1; ähnlich auch Ladstätter u. a. 2003, 24 Taf. 1, K 8.

15 z. B. bei Kenyon 1957, 297 Abb. 68, 10-11.

16 Ähnlich bei Morel 1986, 485 Abb. 27, 149. Von ihm als reduzierend gebrannte Campana C angesprochen, die Beschreibung des Fabrikats erinnert an ephesisches. Das gilt auch für seine Kat. 137, bei welcher auch die Form und der Dekor mit den ephesischen Platten übereinstimmen.

17 Andere bereits bekannte Schalenform aus Ephesos: Meriç 2002, 40-42 Taf. 12, K 123-125. 128 (alle aus Füllung B1 des Brunnens am Staatsmarkt und zeitlich wohl fortgeschrittener als unseres). S. Ladstätter wird aus Hanghaus 2, Wohneinheit 4 ein KatNr. 17 vergleichbares Stück vorstellen: S. Ladstätter, Keramik, in: H. Thür (Hrsg.), Hanghaus 2. Wohneinheit 4. Baubefund, Ausstattung, Funde, FiE VIII 6 (in Druck). 
chen auf Delos ${ }^{18}$, die Qualität des Firnisses und des Scherbens kann aus der Publikation jedoch nicht erschlossen werden. Ein ähnliches Schälchen auf einem ausschwingenden, hellenistisch anmutenden $\mathrm{Fu} ß$ ist aus Ephesos bereits bekannt ${ }^{19}$, ein weiteres mit Roulettierung aus Hanghaus 1 ebenfalls publiziert ${ }^{20}$.

\section{Relevante ephesische Fundkontexte}

Von den bisherigen Fundorten in Ephesos selbst ${ }^{21}$ liefern derzeit der Brunnen am Staatsmarkt ${ }^{22}$, der Brunnen im Raum SR 9c im Hanghaus $1^{23}$ und die hier präsentierten Beispiele von den spätesten hellenistischen Schichten der Tetragonos Agora (sog. Straße II/I, Quaderlage und Raubgruben) über ihre Fundvergesellschaftung und Stratigraphie gute zeitliche Ansätze. Hinzu kommt noch ein Fundkomplex augusteischer Zeit aus Wohneinheit 4 des Hanghauses 2, der demnächst publiziert vorliegen wird ${ }^{24}$.

Für die Produktions- und Benützungszeit in Ephesos können folgende Daten gewonnen werden: Im Brunnen am Staatsmarkt fanden sich alle Beispiele der runden Platten mit niedrigem Rand in der untersten Füllung. Diese Füllung A kann in späthellenistische Zeit datiert werden, mit einer endgültigen Schließung spätestens in augusteischer Zeit. In ihr fehlen Varianten mit höherem gerundetem Rand. Die nachfolgenden Füllungen beinhalten dafür die Platten mit profilierten gerundeten Rändern und vor allem rechteckige Platten (sog. Tabletts). Die hier vorgestellten Schichten der Tetragonos Agora enthalten neben den Beispielen KatNr. 10 und 11 mit hohem gerundetem Rand besonders jene mit niedrigem gerundetem Rand und die aufgelisteten Schüsselbzw. Sonderformen. Die rechteckigen Platten folgen auch hier erst in den Schichten spätaugusteisch-tiberischer Zeit ${ }^{25}$. In Brunnenfüllung 1 im Hanghaus 1 ist neben den zwei Exemplaren mit niedrigem Rand auch noch ein Stück mit etwas höherem gerundetem Rand enthalten ${ }^{26}$. Für die beiden letzten Fundkomplexe wurde eine Datierung in das dritte Viertel, spätestens in das letzte Viertel des 1. Jahrhunderts v. Chr. vorgeschlagen ${ }^{27}$. In allen drei Fundkontexten erwecken die runden Platten den Eindruck von Altstücken, besonders diejenigen mit höherem Rand; auch fehlen in ihnen runde Platten mit niedrigen profilierten Rändern bzw. rechteckige Platten oder Tabletts. Daraus ergibt sich eine weitere relative Abfolge der Formen dieser Gattung.

\section{Außerephesische Fundkontexte - Exportstücke}

Als Exporte konnten bisher hauptsächlich runde Platten mit niedrigem gerundetem Rand und

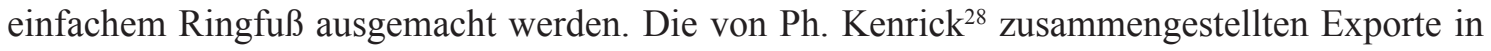

18 Bruneau 1970, 248 Taf. 43, D 56.

19 Mitsopoulos-Leon 1991, 81. 85 Taf. 104, F 60.

20 Outschar 1996, 43 Taf. 12, Kat. 87.

${ }^{21}$ R. Heberdey in: FiE I (1906) 175-176 Nr. 3 vom Rundbau am Panajirdagh; Outschar 1996, 42-44, Kat. $70(?) .71$. 73. 77. 80-85. 90-91 aus Hanghaus 1; Outschar 2000, 142 Abb. 15 Kat. 109-110 von der Hanghausstraße; Zabehlicky-Scheffenegger - Sauer - Schneider 1996, 42-43 Taf. 11, 2. 11; Taf. 18, 57-58 ebenfalls von der Tetragonos Agora, allerdings aus zeitlich späteren Befunden.

22 Meriç 2002, 39 Taf. 11, K 97-100; Taf. 12, K 111; Taf. 17, K 153.

${ }^{23}$ Ladstätter u. a. 2003, 24 Taf. 1, K 8-10.

${ }^{24}$ Ladstätter (Anm. 17) mit Altstücken runder Ephesos-Platten und Schälchen.

25 Dazu Rogl (Anm. 5) 187-188 mit Anm. 3. Dabei ist das Auftreten vereinzelter rechteckiger Platten in Fundkisten der sog. Raubgruben-Befunde aufgrund ungeklärter stratigraphischer Einzelprobleme nicht gänzlich auszuschlieBen, vgl. zwei Beispiele aus Fundkisten EAG 90/46 und EAG 89/82.

26 Ladstätter u. a. 2003, 24 Taf. 1, K 8.

27 Ebenda 26; hier Anm. 5.

28 Bereits Ph. Kenrick erkannte eine Konzentration der Funde auf Samos und in Ephesos und stellte über die Tonzusammensetzung eine Verbindung zu den ionischen Reliefbechern her. 
Berenice, Samos, Delos, Athen, Korinth, Olympia, Çandarli, Priene, Labraunda, Tarsus, Antiochia und Samaria konnten durch S. Zabehlicky-Scheffengger um den Magdalensberg, durch S. I. Rotroff um Paphos, Stobi, Troja, Sardis und Mytilene sowie durch K. Warner Slane um Tel Anafa ergänzt werden ${ }^{29}$. Die zugehörigen Fundkontexte liefern Daten von der Wende des 2. zum 1. Jahrhundert v. Chr. bis in frührömische Zeit. Es zeigt sich jedoch eine Konzentration dieser Plattenform vor der Mitte bzw. um die Mitte des 1. Jahrhunderts v. Chr. Für Platten mit hohem gerundetem Rand konnten nur zwei Orte mit je einem Exemplar ausfindig gemacht werden Berenice und Samos ${ }^{30}$. Die Stücke können als ephesische Produkte angesprochen werden. Für alle weiteren Formen sind außer dem erwähnten Schüsselfragment auf Samos bisher keine exportierten Entsprechungen bekannt.

\section{Vergleiche mit Formen anderer Keramikgattungen}

Die runden Platten mit niedrigem gerundetem Rand und einfachem Ringfuß (KatNr. 1-9) finden Parallelen in den Gattungen der ESA (Hayes 1985, Form Atlante 4A/Slane 1997, Tel Anafa 13c) $)^{31}$, der ESB (Hayes 1985, Form Atlante 18, Prototyp Ephesos) $)^{32}$, der pergamenischen Sigillata $^{33}$, aber auch den italischen Presigillaten ${ }^{34}$ sowie den italischen Gattungen Campana A, B und $\mathrm{C}^{35}$. Vergleiche können auch zwischen den Dekormotiven dieser Gattungen und unseren Beispielen gezogen werden, z. B. die gestempelten Palmetten, die konzentrischen Kreisrillen und die Roulettierungen bei $\mathrm{ESA}^{36}$ oder auch Campana $\mathrm{A}^{37}$ betreffend.

29 Kenrick 1985, 52; Zabehlicky-Scheffenegger - Sauer - Schneider 1996, 42-43. 45 Taf. 18, 57. s. dort auch Taf. 19 mit einer Verbreitungskarte großenteils zu den runden Ephesos-Platten; Rotroff 1997, 233 mit Anm. 60. - Athen: Rotroff 1997, 233. 399 Abb. 75 Nr. 1574-1575 (1574: Kontext 1. Jh. v. Chr.; 1575: 115-86 v. Chr.); Berenice: Kenrick 1985, 53-54 Abb. 9, 71.1-2; 74-75; Delos: Bruneau 1970, 248 Taf. 43, D 53 (Kontext der Zeit Ende 2./Beginn 1. Jh. v. Chr., vgl. hier KatNr. 8); Morel 1986, 482 Abb. 23, 137; Paphos: J. W. Hayes, Paphos III (1991) 8. 151 Abb. 13, 10. 12 (Nr. 22 aus Raum $\Lambda$, Fundkontext mit Keramik der Mitte des 1. Jhs. v. Chr.); Priene: R. Zahn in: Th. Wiegand - H. Schrader, Priene I (1904) 398-399 Abb. 526, 11(-13); Samos: W. Technau, AM 54, 1929, 48 Beil. 28, 1-2; Tel Anafa: Slane 1997, 369-370 Taf. 32, FW 545-546 (aus frühen römischen Horizonten, erscheinen jedoch dort als Altstücke). - Die Kontexte von Korinth enthalten auch spätere Plattenformen und entstammen tiberischer Zeit. Neu hinzugetreten ist Sardis. Vgl. S. I. Rotroff - A. Oliver, The Hellenistic pottery from Sardis: The finds through 1994, Sardis Monograph XII (2003) 32-33 Taf. 16; 83-91 mit dem interessanten Hinweis auf eine mögliche lokale Produktion dieser Platten in Sardis.

${ }^{30}$ Kenrick 1985, 56 Abb. 9, 76. Durch Analyse des Scherbens als 'Black-glazed C²-ware' (Ionien) identifiziert; das Stück auf Samos wurde mir durch S. Morhoff bekannt.

31 Kenyon 1957, 291 Abb. 65, 3 (Samaria, Form 1); Mitsopulos-Leon 1991, Taf. 105, G 1-2; weitere unveröffentlichte Beispiele von der Tetragonos Agora, Inv. AG 89/16-14 und 89/16-15; Ladstätter u. a. 2003, 29 Taf. 7, K 68-69c aus Brunnenfüllung 2 (um 100 v. Chr.); 23 Taf. 1, K 1-2. 5 aus Brunnenfüllung 1 (3. Viertel 1. Jh. v. Chr.); Bruneau 1970, 245-246 Abb. 126, D 43-45; F. Queyrel in: R. Etienne (Hrsg.), Ténos I. Le sanctuaire de Poseidon et d'Amphitrite (1986) 222 Taf. 121, Eb 2 aus Gruppe Eb mit Keramik aus der Zeit Mitte bzw. 3. Viertel 1. Jh. v. Chr. und mit Vergleich zur Campana, s. Morel 1986; aber auch reduzierend gebrannte ESA-Teller auf Delos (Bruneau 1970, 249 Taf. 43, D 64) bzw. in Tel Anafa (Slane 1997, 364 mit Verweis auf J. W. Hayes und reduzierend gebrannte zyprische Sigillata).

32 Ladstätter u. a. 2003, 24 Taf. 1, K 7 mit Anm. 24 zur Diskussion um die Zusammenhänge von ESA-, ESB- und pergamenischer Sigillataproduktion. Dazu auch Meriç 2002, 40 mit den Schalenformen dieser Gattungen. Bruneau 1970, D 50-52.

33 Meyer-Schlichtmann 1988, Taf. 32, T 2 (Teller mit Roulettierung).

34 Stone 1987, 101 Abb. 16, $48-50$ (tritt ab dem 4. Viertel des 1. Jhs. v. Chr. auf).

35 Morel 1986, 464 Abb. 3, 14-15 (Campana A); ebenda 473 Abb. 15.18 (Campana B); ebenda 478 Abb. 19 (Campana C).

36 Vgl. Mitsopulos-Leon 1991, 80 mit Anm. 415-417; Taf. 115, G 32-35; Mitsopoulos-Leon 1975, Taf. 27-30; Kenyon 1957, 293 Abb. 66; 317 Abb. 74; 320 Abb. 75.

37 Morel 1986, 467 Abb. 8. 
Die Form des halbkugeligen Schälchens (KatNr. 17) entspricht den bekannten frühen ESASchälchen (Hayes 1985, Atlante Form 22B/Slane 1997, Tel Anafa 25) ${ }^{38}$, findet sich aber auch in ESB-Qualität (Hayes 1985, Atlante Form 29, Prototyp Ephesos) und dort sogar in reduzierend gebranntem Scherben ${ }^{39}$. Auch hier kann die Liste der Vergleiche mit pergamenischer Sigillata, italischer Presigillata und wiederum Camapana A fortgesetzt werden ${ }^{40}$.

Für die runden Platten mit hohem, außen gerilltem Rand oder Schüsseln (KatNr. 12) findet sich kein wirkliches Vergleichsbeispiel. Eine einzige ähnliche Form in Qualität der CampanaC-Keramik wurde für Delos vorgestellt ${ }^{41}$.

Die ältesten bereits immer in Kombination erscheinenden Keramikformen innerhalb der sog. Ostsigillaten - die Platten bzw. flachen Teller und die halbkugelförmigen Schälchen - stehen noch in der Tradition hellenistischer Gefäß $\mathrm{e}^{42}$. Dies deuten der runde Wandverlauf und die breiten, oft ausschwingenden Füße an; aber auch der Dekor mit Palmettenstempel, Rosetten und Isiskronen in meist zentraler bzw. konzentrischer Anordnung führt dies weiter. Doch zeigen die Ostsigillaten eine vollkommen neue Gesinnung in ihrer Gewichtung auf die zwei Hauptformen Platte und halbkugelförmiges Schälchen. Diese Formen finden sich nicht nur in Ephesos wie im gesamten Osten des Reiches, sondern in ähnlichen oder abweichenden Qualitäten und unterschiedlichen Quantitäten im gesamten Mittelmeerraum.

$\mathrm{Ab}$ augusteischer Zeit und unter Einfluß der westlichen Keramikgattungen werden auch die Formteile der Ostsigillaten schärfer voneinander abgesetzt. Der Formenreichtum an sich nimmt $\mathrm{zu}$, und die üblichen Dekorstempel wie auch die Roulettierung entfallen: sie werden durch Rechteckstempel mit Namen und Grußstempel ersetz ${ }^{43}$.

Eine weitere wichtige Beobachtung bei der Bearbeitung der frühen Sigillaten ist der Nachweis reduzierend gebrannter Exemplare innerhalb der $\mathrm{ESA}^{44}$, der $\mathrm{ESB}^{45}$, aber auch der frühen italischen bzw. lokalen Sigillaten ${ }^{46}$, wie wir es bereits von den hellenistischen Gattungen wie den Reliefbechern, Lampen, Medaillonschalen, knidischen Schalen mit Appliken- und/oder Stempeldekor oder einzelnen Gefäßen mit Dekor im sog. Westabhang-Nachfolgestil und den Terrakotten kennen $^{47}$. Diese Feststellung wurde bereits an vielen Fundplätzen gemacht, ohne jedoch dabei Konsequenzen in der Zuordnung der grauen oder schwarzen Keramik zu ziehen ${ }^{48}$. So finden sich

38 Kenyon 1957, 291 Abb. 65, 1-2 (Samaria, Form 16); Bruneau 1970, 245-246 Abb. 126, D $46-47$ und D 52; Ladstätter u. a. 2003, 23 Taf. 1, K 3(?). K 4b-c.

39 Outschar 1996, 43 Taf. 4, Kat. 79.

40 Meyer-Schlichtmann 1988, Taf. 30, N 2. N 3; Stone 1987, 98 Abb. 11, 34; Morel 1986, 466 Abb. 7, 30-31. Ganz allgemein muß hier natürlich auch auf die späthellenistischen Echinusschälchen als ähnliche Form hingewiesen werden. Diese besitzen allerdings meist steilere Wandungen und stärker eingebogene Lippen.

${ }^{41}$ Vgl. o. Anm. 16.

42 Dazu auch Kenyon 1957, 355-357 (einschließlich einer Diskussion um das Entstehungszentrum).

43 Mitsopoulos-Leon 1991, 86-87 mit Anm. 455-458; dies. 1975, 110. Eine ähnliche Entwicklungslinie im keramischen Bereich kann auch im westlichen Mittelmeerraum von der sog. Campana C über die sog. Presigillata hin zur Arretina nachvollzogen werden. Vgl. Stone 1987, 85. 91.103 (Qualität, Datierung); 92-102 (Formen) mit Verweisen auf die Arbeiten von N. Lamboglia und C. Goudineau.

44 Vgl. ein reduzierend gebranntes ESA-Schälchen (Inv. AG 00/63-1) aus den Grabungen des Jahres 2000 im Nordtorbereich der Tetragonos Agora von Ephesos. Es entspricht Hayes 1985, Form 11, allerdings mit höherem geschwungenem Fuß und kann anhand chemischer und petrographischer Untersuchungen als »in der Region von Ephesos hergestellt« angesprochen werden.

45 S. Ladstätter, Rei Cretariae Romanae Fautorum Acta 36, 2000, 102-103 Abb. 7, 3; Outschar 1996, 43 Taf. 4 , Kat. 79.

46 M. Schindler, Die 'Schwarze Sigillata' des Magdalensbergs, Kärntner Museumsschriften 43 (1967). Datierung um die Mitte des 1. Jhs. v. Chr., wohl Vorformen zur roten Sigillata, s. dort S. 65. 67.

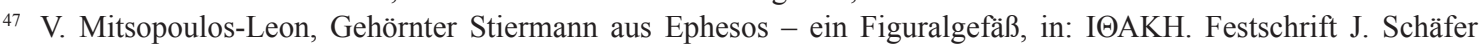
(2001) 219-224. Reduzierend gebrannte Beispiele verschiedenster Keramikgattungen lassen sich bis in die Bronzezeit zurückverfolgen. Im beginnenden 1. Jh. v. Chr. scheint es jedoch eine besondere Vorliebe dafür im gesamten Mittelmeerraum zu geben.

48 Mitsopoulos-Leon 1991, 78 mit Anm. 392-397 und einer Diskussion zu einzelnen Fundorten. s. auch Rotroff 1997, 232 mit Anm. 57. 
die graugebrannten Exemplare vieler Gattungen meist getrennt von ihren oxidierend gebrannten Entsprechungen, werden aber in den jeweiligen Grabungspublikationen meist gattungsvermischt in einem der 'Grauen Keramik' gewidmeten Kapitel behandelt. In nur wenigen Fällen geschah dies auch mit guten Begründungen. So trennte K. W. Slane die graue zyprische Sigillata von ihren roten Entsprechungen, da sie ihnen zeitlich voranzugehen scheint ${ }^{49}$. Sollte eine ähnliche Überlegung auch für die früheste schwarze ephesische Sigillata in Erwägung gezogen werden oder ein zeitliches Nebeneinander der frühesten ESA-Importe und der lokalen Produktion der grauen Entsprechungen? Zukünftige Befunde werden - nach unserer Erörterung der Problematik - hoffentlich Klärung bringen.

\section{Servicerepertoire und mögliche Veränderungen der Tisch- und Eßkultur}

Bleibt die Frage der Verwendung und der Kombination unserer Platten bzw. Einzelformen bei Tisch. Auffallend ist, daß sich im Vergleich zu den zahlreichen Plattenfragmenten kaum Fragmente von Schälchen finden. Auch J.-P. Morel stieß bei seiner Untersuchung zur CampanaB-Keramik auf Delos auf diese Problematik ${ }^{50}$ : Den zahlreichen Tellern dieser Keramikgattung konnten ebenfalls keine Trinkgefäße zugeordnet werden. So stellte er die Frage, ob die Teller mit Metallbechern kombiniert wurden, oder die lokalen Reliefbecher aufgrund eines langen Nachlebens diese Rolle übernahmen. Der Großteil der Campana-B-Importe konnte von ihm in die Zeit des zweiten Viertels des 1. Jahrhunderts v. Chr. datiert werden ${ }^{51}$. Diese Datierung könnte sich mit jener der Herstellung der frühesten grauen Platten in Ephesos decken.

In dieselbe Periode fallen sowohl die Produktionszeiten applikenverzierter Trinkgefäße als auch solcher mit Dekor im sog. Westabhang-Nachfolgestil. Sie konnten reduzierend oder oxidierend gebrannt, beide Verfahren jedoch auch an einem Gefäß angewandt $\operatorname{sein}^{52}$. Auch weisen beide Gattungen erstmals glänzenden sigillataartigen Überzug auf. Spricht man sie als Tafelgeschirr an, so würden bei ihnen zugehörige Teller oder ähnliche Formen fehlen ${ }^{53}$. Fanden sie demnach nur bei Symposien Verwendung? Wurden sie mit Tellern, wie z. B. unseren Platten, kombiniert? Die Beantwortung dieser Fragen fällt derzeit schwer. Und zeigen alle hier erwähnten Gefäßformen nicht auch einen Übergang von griechisch-hellenistischem Tafel- und Trinkgeschirr zu römisch-italischen Formen? Kündigt sich hier nicht eine Änderung der Gewohnheiten bzw. Sitten bei Tisch an?

Somit wird mit der Betrachtung der frühen schwarzen ephesischen Sigillata auch eine noch nicht näher zu definierende Änderung der Tischsitten in einer Zeit des politischen und gesellschaftlichen Umbruchs im Osten des Reiches um die Mitte des 1. Jahrhunderts v. Chr. greifbar ${ }^{54}$.

49 Slane 1997, 366.

50 Morel 1986, 490. Seine Beobachtung, ebenda 487, daß Delos der einzige Fundplatz im Osten ist, an welchem Campana-Keramik in beachtenswerter Zahl auftritt, scheint sich zu bestätigen. Im Gegensatz zu Delos fand sich nach Durchsicht Hunderter Fundkisten aus entsprechenden Befunden in Ephesos kaum Campana-Keramik. Die umfangreiche lokale Produktion in und um Ephesos und ihr Umstieg auf die neuen Formen in der Qualität unserer grauen Platten scheint einen Import überflüssig gemacht zu haben.

51 Morel 1986, 489-490 (nach 88 v. Chr. wahrscheinlich noch importiert, aber auch nach 69 v. Chr.?).

52 Innen oxidierend und außen reduzierend. Reduzierend gebrannte Beispiele aus Ephesos finden sich z. B. bei Meriç 2002, Taf. 12, K 126-127 mit Westabhang-Nachfolgedekor und K 128 mit Applikendekor, auch K 125. Alle stammen aus Füllung B1 und könnten im Gegensatz zu den flachen Schälchen K 123-124 aufgrund ihrer Kleinteiligkeit als Altstücke in dieser Füllung angesprochen werden.

53 Im Bereich der einfachen Firniskeramik sind sowohl Teller als auch Schalen vorhanden, besonders Teller mit gedrechseltem Rand bzw. mit breiter Lippe und Echinusschalen mit steiler Wandung bzw. mit Wandknick und ausgebogener Lippe. Vgl. Ladstätter u. a. 2003, 24, K 11-14; 32-34, K 74-88. 95-101. 111-158. 162. 163. 172176.

${ }^{54}$ Ich danke S. Ladstätter und A. Giuliani für fachliche Diskussion und Hinweise. N. Math sei für die Erstellung der Tafeln gedankt. 


\section{Katalog}

KatNr. 1 (Abb. 1 Taf. 1, K 1)

Inv. AG 91/10-10

Form: Platte, rund, niedriger Rand

DmR 30,0 cm; H 1,7 cm; B 6,0 cm; Ws 0,45-0,5 cm Ton: 7.5YR-4/4 dark brown; hart; fein, porös, Glimmer Firnis: 7.5YR-N2/0 black 4/1 (schwarz); leicht glänzend; haftet gut

Erhaltung: Rand/Boden.

KatNr. 2 (Taf. 1, K 2)

Inv. AG 93/68-1

Form: Platte, rund, niedriger Rand

DmR 30,2 cm; H 2,1 cm; B 8,7 cm; Ws $0,5 \mathrm{~cm}$

Ton: $2.5 \mathrm{Y}-5 / 2$ grayish brown (grau); mittelhart; mit dunklen und weißen Beimischungen, Glimmer

Firnis: a: schwarz 2.5Y-3/1 very dark gray (schwarz), i: 10YR-4/2 dark grayish brown (braun); leicht glänzend; teils berieben; haftet gut

Erhaltung: Rand/Boden.

KatNr. 3 (Taf. 1, K 3)

Inv. AG 89/15-7

Form: Platte, rund, niedriger Rand

DmR $32 \mathrm{~cm}$; H 2,3 cm; B 11,3 cm; Ws 0,45-1,3 cm

Ton: braungrau; mittelhart; porös, wenig Glimmer

Firnis: schwarz; glänzend; gut haftend

Erhaltung: Rand/Bodenansatz.

KatNr. 4 (Taf. 1, K 4)

Inv. AG 90/13-14

Form: Platte, rund, niedriger Rand

DmR 29,0 cm; H 2,2 cm; B 11,0 cm; Ws 0,7 cm

Ton: 10YR-5/2 grayish brown (a: grau, i: braun); sehr hart; porös, kleine weiße Beimischungen, Glimmer

Firnis: 2.5YR-N3/very dark gray (schwarz); matt und teilweise glänzend; teils abgerieben

Erhaltung: Rand/Boden.

KatNr. 5 (Taf. 1, K 5)

Inv. AG 89/16-11

Form: Platte, rund, niedriger Rand

DmR 36,8 cm; H 2,2 cm; B 13,6 cm; Ws 0,5-0,6 cm

Ton: braugrau; mittelhart; fein, Glimmer

Firnis: schwarz; glänzend; haftet gut

Erhaltung: Boden

Dekor: Dekorband mit Roulettierung - Zone mit Palmettenstempel - Reste eines weiteren Dekorbandes mit Roulettierung.

KatNr. 6 (Taf. 1, K 6)

Inv. AG 93/32-5

Form: Platte, rund, niedriger Rand

DmR 25,4; DmB 8,8 cm; H 3,5 cm; B 16,0 cm; Ws $0,6-0,7 \mathrm{~cm}$

Ton: 10YR-5/2 grayish brown (grau); sehr hart; fein; weiße Beimischungen, Glimmer

Firnis: 7.5YR-N3 very dark gray (schwarz); glänzend, sigillataartig, gut haftend; a: berieben Erhaltung: vollständiges Profil
Dekor: Zwei zentrale Kreisrillen innen - vier eingestempelte stilisierte Palmettenmotive - zwei Kreisrillen - feine Roulettierung - zwei Kreisrillen.

KatNr. 7 (Abb. 2 Taf. 1, K 7)

Inv. AG 96/47-26

Form: Platte, rund

DmB 10,0 cm; H 1,3 cm; B 13,3 cm; Ws 0,5 cm

Ton: 10YR-5/2 grayish brown (braungrau); hart; fein; kleinste weiße Beimischungen, Glimmer

Firnis: 7.5YR-N3 very dark gray (schwarz); glänzend poliert, gut haftend; um den Standring nicht gefirnißt Erhaltung: Standring/Boden

Dekor: Zwei zentrale Kreisrillen innen - vier eingestempelte stilisierte Blattpalmetten - zwei Kreisrillen - feine Roulettierung - zwei Kreisrillen.

KatNr. 8 (Taf. 1, K 8)

Inv. AG 90/13-1

Form: Platte, rund

DmB 8,0 cm; H 2,0 cm; B 8,6 cm; Ws 0,6-0,8 cm

Ton: grau; hart; fein, Glimmer

Firnis: i: schwarz; matt, da berieben; a: tongrundig

Erhaltung: Boden/Standring

Dekor: Spitze eines Palmettenstempels, liegt auf einem Dekorband mit Roulettierung.

KatNr. 9 (Taf. 1, K 9)

Inv. AG 92/50-18

Form: Platte, rund

DmB ca. $10 \mathrm{~cm}$; H 1,5 cm; B 5,4 cm; Ws 0,6-1,0 cm

Ton: (grau)braun; mittelhart; fein, porös, Glimmer

Firnis: schwarz; glänzend; teils abgerieben

Erhaltung: Boden

Dekor: Dekorband mit Roulettierung - Zone mit Palmettenstempel - Reste eines weiteren Dekorbandes mit Roulettierung.

KatNr. 10 (Abb. 3 Taf. 2, K 10)

Inv. AG 89/16-12

Form: Platte, rund, hoher Rand

DmR 26,0 cm; H 4,7 cm; B 7,2 cm; Ws 0,7-0,8 cm

Ton: grau; mittelhart; feine weiße Beimischungen, Glimmer

Firnis: schwarz; glänzend; haftet gut

Erhaltung: Rand/Boden.

KatNr. 11 (Taf. 2, K 11)

Inv. AG 89/15-6

Form: Platte, rund, hoher Rand

DmR $34 \mathrm{~cm}$; H 4,9 cm; B 7,9 cm; Ws 0,45-1,2 cm

Ton: bräunlich grau; mittelhart; wenig Glimmer

Firnis: schwarz; glänzend; gut haftend

Erhaltung: Rand/Boden.

KatNr. 12 (Abb. 4 Taf. 2, K 12)

Inv. AG 89/15-8

Form: Platte, rund, mit außen gerilltem Rand oder Schüssel 

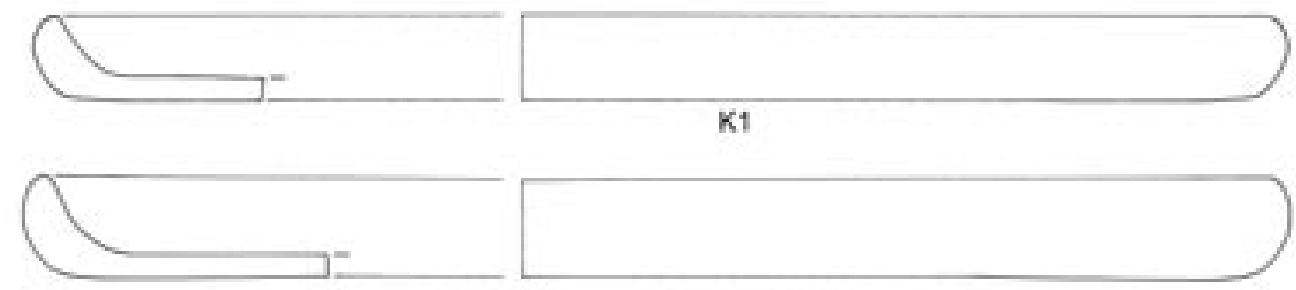

K2
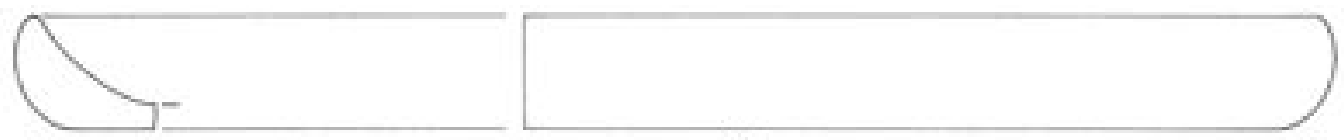

$\mathrm{K} 3$
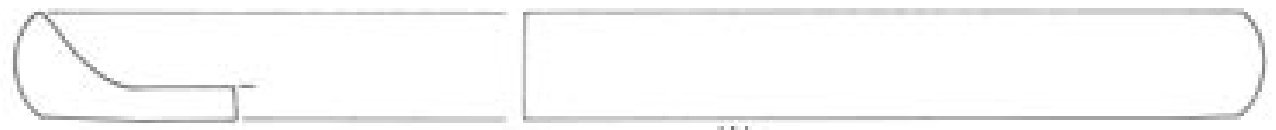

K4
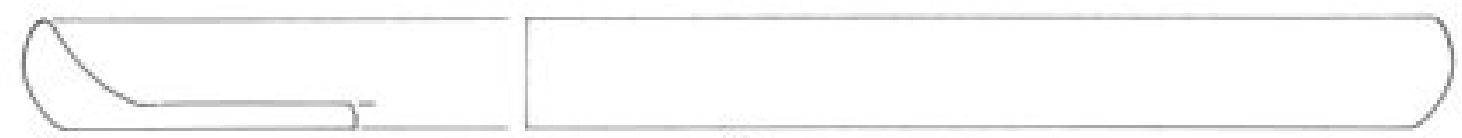

K5
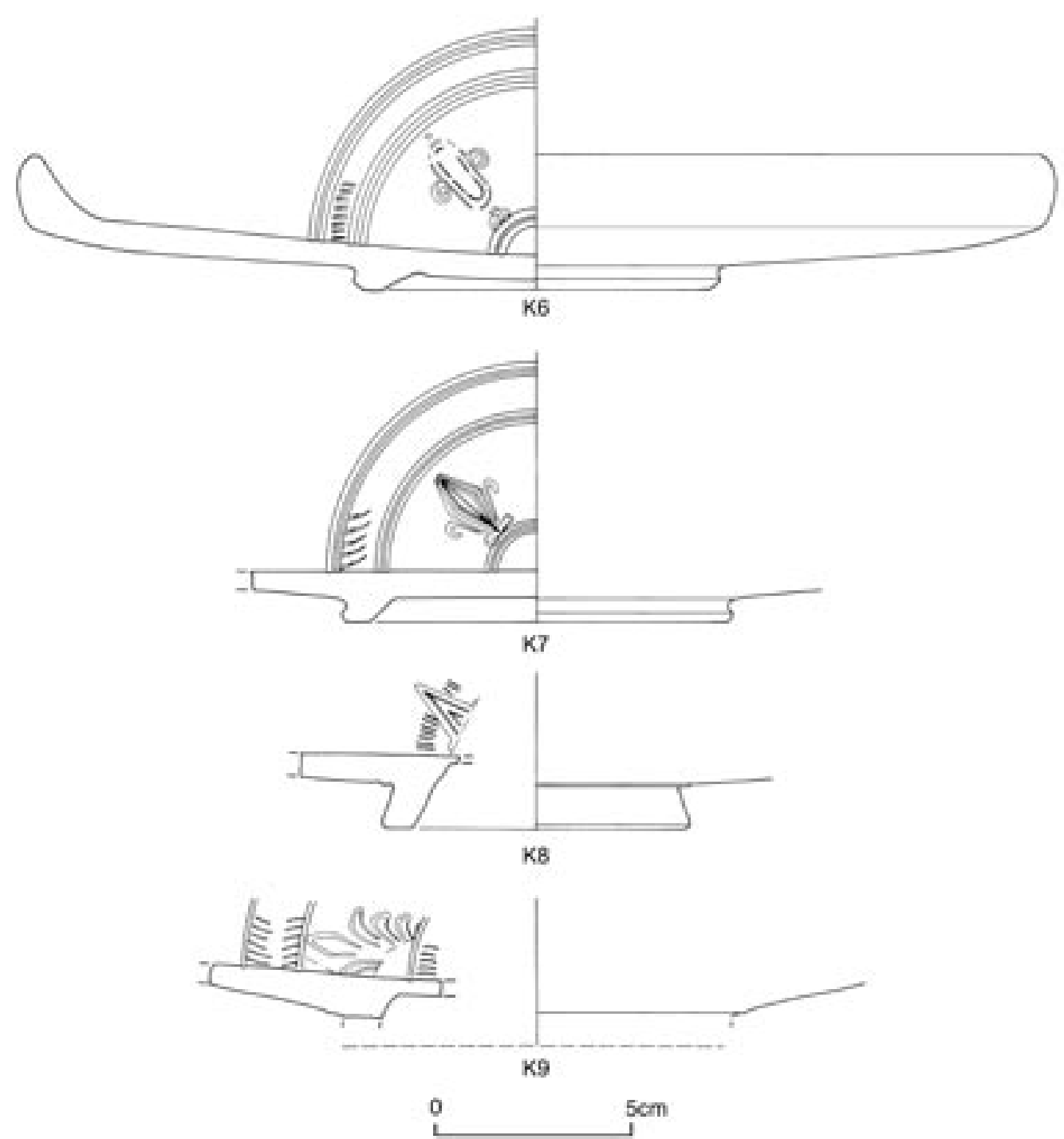

Taf. 1 Runde Platten der frühen schwarzen Sigillata aus Ephesos. Beispiele aus den Grabungen auf der Tetragonos Agora 

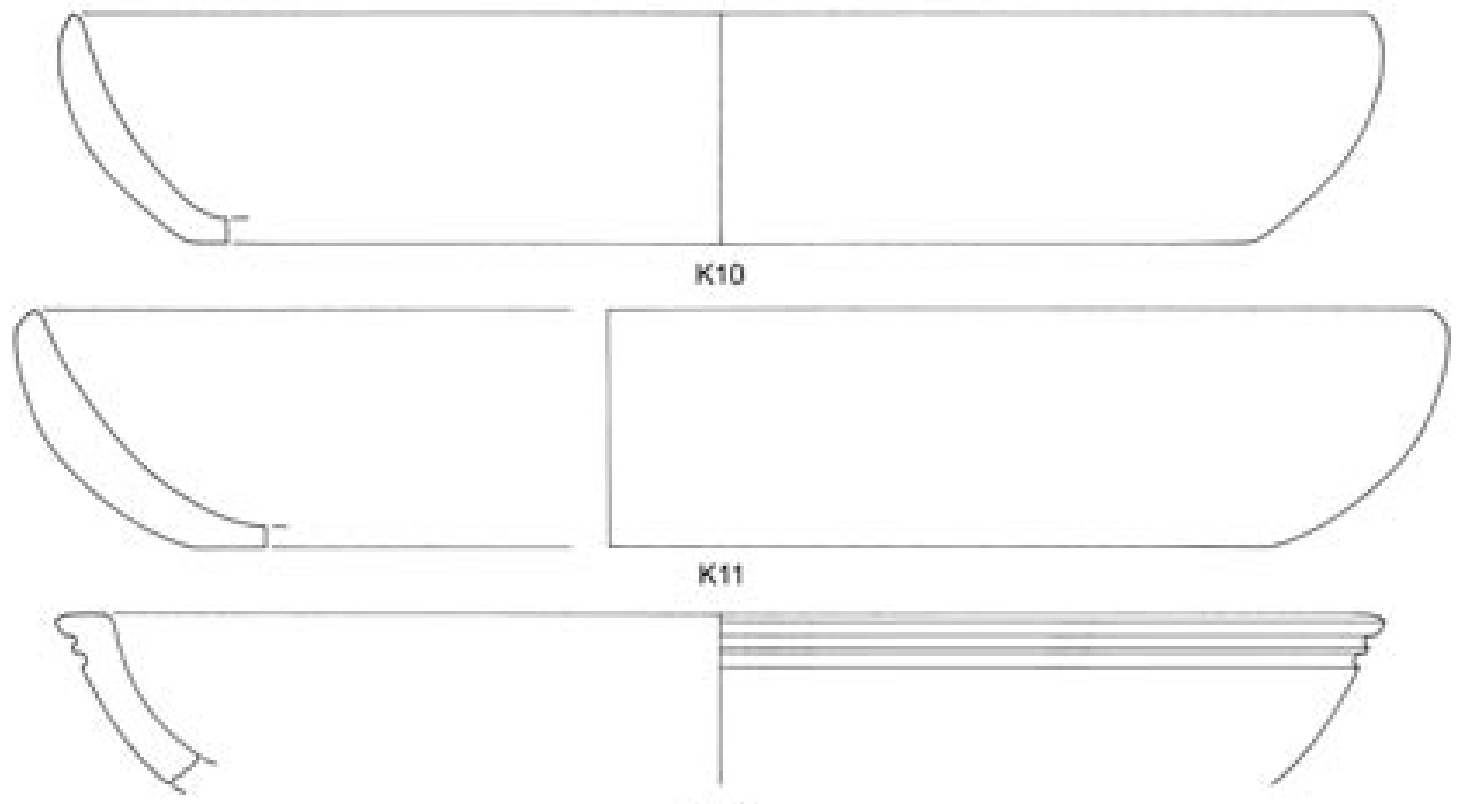

K12
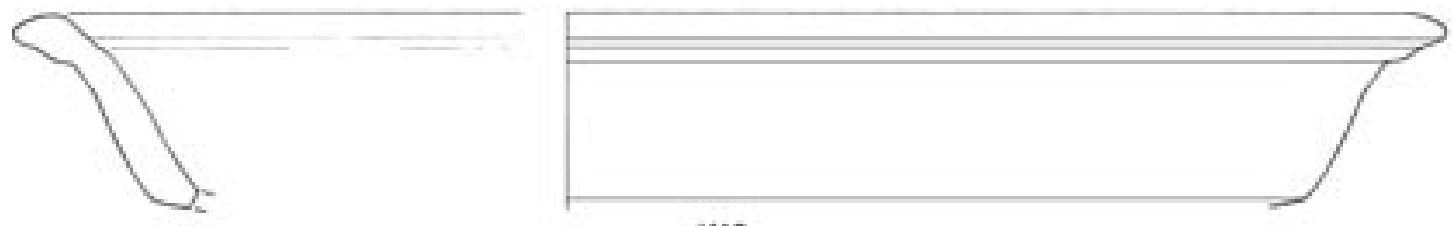

K13
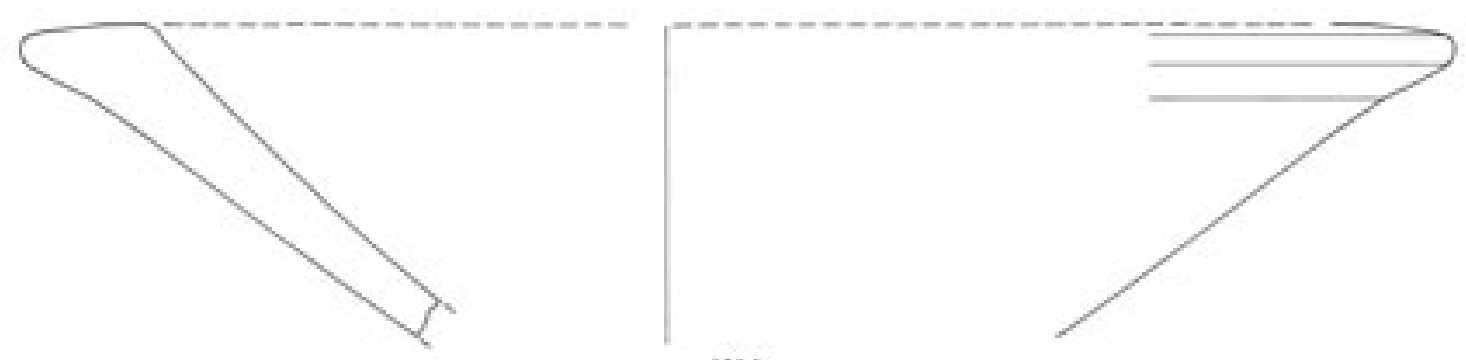

K14
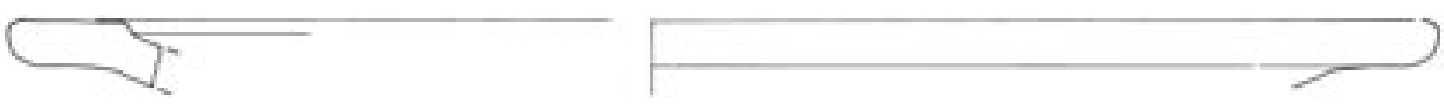

K15
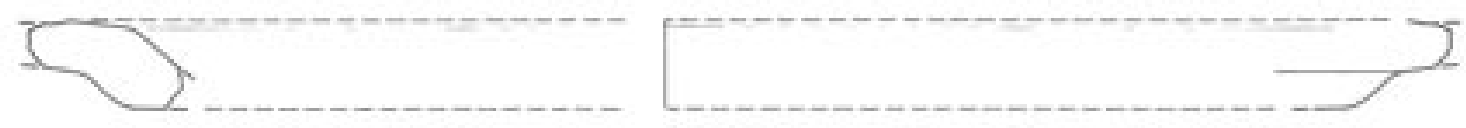

K16

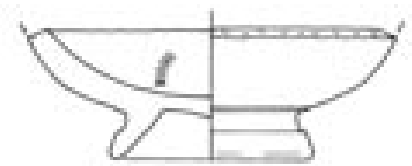

K17

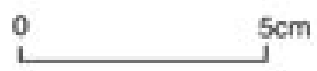

Taf. 2 Runde Platten, Schüsseln und Schale der frühen schwarzen Sigillata aus Ephesos. Beispiele aus den Grabungen auf der Tetragonos Agora 
DmR 27,0 cm; H 3,4 cm; B 6,6 cm; Ws 0,8-1,1 cm Ton: braungrau; mittelhart; porös, wenig Glimmer Firnis: schwarz; glänzend; gut haftend Erhaltung: Rand.

KatNr. 13 (Taf. 2, K 13)

Inv. AG 93/32-2

Form: runde Platte oder Schüssel mit hohem ausschwingendem Rand

DmR 34,4 cm; H 4,0 cm; B 7,5 cm; Ws 0,7-0,9 cm

Ton: 10YR-5/2 grayish brown (grau, a: bräunlich); sehr hart; fein, porös, kleine weiße Beimischungen, Glimmer Firnis: 10YR-3/1 very dark gray (schwarz); eher matt; etwas berieben

Erhaltung: Rand/Wand.

KatNr. 14 (Taf. 2, K 14)

Inv. AG 93/32-4

Form: tiefe Schüssel mit hohem, weitausladendem massivem Rand

DmR ca. 50,0 cm; H 6,3 cm; B 16,0 cm; Ws 0,8 cm (am Rand verdickt)

Ton: 10YR-6/2 bzw. 5/2 light brownish gray bzw. grayish brown (i: grau, a: braun); hart; fein, porös, kleine weiße Beimischungen, Glimmer

Firnis: 10 YR-4/1 bzw. 3/1 very dark gray (schwarz bis dunkelgrau); matt bis leicht glänzend; berieben Erhaltung: Rand/Wand.
KatNr. 15 (Taf. 2, K 15)

Inv. AG 93/48-5 (Straße II/I)

Form: Platte, rund, mit horizontal abgesetztem Rand

DmR ca. $30-38 \mathrm{~cm}$; H 1,4 cm; B 4,3 cm; Ws $0,8 \mathrm{~cm}$

Ton: Gley N4 dark gray (grau); sehr hart; gröbere helle Beimischungen, Glimmer

Firnis: nur teilweise erhalten, da Oberfläche verbrannt; Gley N5 gray (schwarz)

Erhaltung: Rand.

KatNr. 16 (Taf. 2, K 16)

Inv. AG 93/69-3 (Quaderlage)

Form: Platte, rund, mit horizontalem Rand

$\mathrm{DmB} / \mathrm{R}$ ?; H $1,8 \mathrm{~cm}$; B 8,3 cm; Ws ca. $0,8 \mathrm{~cm}$

Ton: grau; mittelhart; fein, porös, wenige weiße und dunkle Beimischungen, viel Glimmer

Firnis: schwarz; glänzend; stark berieben

Erhaltung: Rand beschlagen.

KatNr. 17 (Taf. 2, K 17)

Inv. AG 93/32-3

Form: halbkugeliges Schälchen auf Ringfuß

DmB 4,2 cm; H 2,6 cm; B 6,8 cm; Ws 0,3-0,6 cm

Ton: $10 \mathrm{YR}-3 / 2$ very dark grayish brown (graubraun); hart; fein, viel Glimmer

Firnis: 2.5YR-N3 very dark gray (schwarz); matt bis leicht glänzend, sigillataartig; berieben

Erhaltung: Fuß/Boden

Dekor: Feine kreisförmige Roulettierung innen am Schalenboden.

\section{Abkürzungen und abgekürzt zitierte Literatur}

$\begin{array}{llll}\text { a } & \text { außen } & \text { ESA } & \text { Eastern Sigillata A } \\ \text { AG } & \text { Tetragonos Agora } & \text { ESB } & \text { Eastern Sigillata B } \\ \text { DmB } & \text { Durchmesser/Boden } & \text { i } & \text { innen } \\ \text { DmR } & \text { Durchmesser/Rand } & \text { Ws } & \text { Wandstärke }\end{array}$

Bruneau 1970

Hayes 1985

Kenrick 1985

Kenyon 1957

Ladstätter u. a. 2003

Meriç 2002

Meyer-Schlichtmann 1988

Mitsopoulos-Leon 1975

Mitsopoulos-Leon 1991

Morel 1986
Ph. Bruneau (Hrsg.), L'îlot de la Maison des Comédiens, Délos XXVII (1970).

J. W. Hayes, Sigillate Orientali, in: EAA II. Ceramica fine Romana nel bacino Mediterraneo (1985) 9-96.

Ph. Kenrick, Excavations at Sidi Khrebish, Benghazi (Berenice) III 1: The Fine Pottery, 5. Suppl. LibyaAnt (1985).

K. M. Kenyon in: J. W. Crowfoot - G. M. Crowfoot - K. M. Kenyon, The objects from Samaria III (1957).

S. Ladstätter - Ch. Rogl - A. Giuliani - T. Bezeczky - B. Czurda, Keramik. Ein hellenistischer Brunnen in SR 9C, in: C. Lang-Auinger (Hrsg.), Hanghaus 1 in Ephesos. Funde und Ausstattung, FiE VIII 4 (2003) 22-85.

R. Meriç, Späthellenistisch-römische Keramik und Kleinfunde aus einem Schachtbrunnen am Staatsmarkt in Ephesos, FiE IX 3 (2002).

C. Meyer-Schlichtmann, Die pergamenische Sigillata aus der Stadtgrabung von Pergamon, PF 6 (1988).

V. Mitsopoulos-Leon, Gefäße der Ostsigillata A von einem gesunkenen Schiff aus dem Golf von Antalya, Germania 53, 1975, 101-112.

V. Mitsopoulos-Leon, Die Basilika am Staatsmarkt in Ephesos. Kleinfunde. 1. Teil: Keramik hellenistischer und römischer Zeit, FiE IX 2, 2 (1991).

J.-P. Morel, Céramiques à vernis noir d'Italie trouvées à Délos, BCH 110, 1986, 461-493. 
Outschar 1996

Outschar 2000

Rotroff 1997

Slane 1997

Stone 1987

Zabehlicky-Scheffenegger Sauer - Schneider 1996
U. Outschar in: C. Lang-Auinger, Hanghaus 1 in Ephesos. Der Baubefund, FiE VIII 3 (1996) 27-85.

U. Outschar, Keramik macht Baugeschichte, RömHistMitt 42, 2000, 106-169.

S. I. Rotroff, Hellenistic pottery. Athenian and imported wheelmade table ware and related material, Agora XXIX (1997).

K. W. Slane, The Fine Wares, in: S. C. Herbert (Hrsg.), Tel Anafa II, 1. The Hellenistic and Roman Pottery, 10. Suppl. JRA (1997) 247-346.

S. C. Stone, Presigillata from Morgantina, AJA 91, 1987, 85-103.

S. Zabehlicky-Scheffenegger - R. Sauer - G. Schneider, Graue Platten aus Ephesos und vom Magdalensberg, in: M. Herfort-Koch - U. Mandel - U. Schädler (Hrsg.), Hellenistische und kaiserzeitliche Keramik des östlichen Mittelmeergebietes, Kolloquium Frankfurt 24.-25.April 1995 (1996) 41-59.

Dr. Christine Rogl

Institut für Kulturgeschichte der Antike, Österreichische Akademie der Wissenschaften, Bäckerstraße 13, A-1010 Wien

E-Mail: christine.rogl@oeaw.ac.at

Abbildungsnachweis: Taf. 1-2: Zeichnung Verf., graphische Gestaltung N. Math (ÖAW); Abb. 1. 3. 4: (C) ÖAI Wien, Photo Verf.; Abb. 2: (C) ÖAI Wien, Photo N. Gail. 
